\title{
Editorial
}

\section{Contributions by researchers of "Ciencia de Frontera" of the Chilean Academy of Sciences}

The aim of this special issue of Biological Research is to present some of the latest advances in biological research of scientists under the Frontiers of Science Program of the Chilean Academy of Sciences of the Instituto de Chile.

This program started 7 years ago, with the idea of incorporating into the Academy the very best young scientists working in Chile in order to discuss exciting advances and opportunities in their fields in a format that encourages informal collective, as well as one-on-one, discussions among participants. Several symposia have been organized over the years, and in each case speakers have been urged to focus their talks on describing current cutting-edge research in their disciplines to colleagues outside their field and to address questions such as: "What are the major research problems and distinctive tools of your field?" and "How might insights derived from other fields contribute to overcoming these limitations?" Participants remain active in the Frontiers of Science Program for an average of 3 years. Such groups have included leading researchers in disciplines such as astronomy, biology, chemistry, geology, mathematical sciences and physics. Here the Society of Biology has invited biologists that have been part of Frontiers of Science Groups to present their latest and most exciting work to the readers of Biological Research.

In the first study, Allende's team describes how a teleost, the zebrafish, can be useful to study the cellular and molecular mechanisms associated with copper toxicity in water, establishing a framework for further analyses of copper excess using an entire organism. The use of invertebrate models for research is also presented by Glavic et al. In this work, they describe how the stability of the Notch protein is dependent on the activity of GDP-mannose-dehydratase (GMD), and therefore on the levels of O-fucose moieties added to the Notch extracellular domain. These post-translational modifications will ultimately contribute to regulate the endocytosis and degradation of Notch.

The usefulness of vertebrate models for research is discussed by several papers describing immunology, cell biology, neurobiology and development. Thus, during the development of the nervous system morphogens are responsible for the establishment of neuronal territories. Palma, Larrain et al describe how the extracellular matrix may modulate the function of the morphogen Sonic Hedgehog $(\mathrm{SHH})$. They specifically propose perlecan as the proteoglycan involved in both $\mathrm{SHH}$ localization and activity, through a direct interaction among these molecules. After the establishment of neural territories, several cellular events lead to neuronal differentiation. Borquez and GonzalezBillault analyze the molecular mechanisms contributing to the establishing of cell polarity. This review presents evidence relating the ubiquitin-proteosome and calpain systems, with cell migration and the development of an axon in neuronal cells, two processes that require the polarization of cells. Another interesting aspect of neuronal function is described in the work of Couve's team. These authors review the state-of-the-art in studying the endoplasmic reticulum in neuronal cells, providing a detailed analysis of its structure, composition, dendritic distribution and dynamics. A functional link is provided with the analysis of the possible roles for the endoplasmic reticulum in synaptic transmission. In the immunological system, dendritic cells (DC) are responsible for maintaining adaptive immunity because they can modulate T-cell activities. Kalergis' team present a review of the interactions between dendritic and T-cells. Consequences of altered DC-T cells are analyzed in the context of autoimmune diseases. Pathological conditions are also presented in the paper by Marzolo and Farfan. This article deals with the role of megalin, a receptor for apolipoprotein E that is implicated in several diseases such as Lowe Syndrome, Dent Disease, Alzheimer's disease and gallstone disease. The authors review the current known functions and propose mechanisms that control megalin expression.

Vegetal biology is also represented by the work of Moreno and Orellana, which describes the plant unfolded protein response (UPR). This is an up-to-date review showing the emergence of plant homologues for UPR proteins, and proposes some mechanistic linkage to physiological responses in plants.

Nature biologists are also represented in this special issue by three articles. Nespolo`s team, analyze how morphological changes occurring during ontogeny may alter the metabolism of invertebrates. Metabolism in vertebrates is analyzed by Ramirez-Otarola and Sabat. They present an article showing how dietary habits may influence the physiology of digestion in eight bird species. Changes in the enzymatic activities amongst species led the authors to propose a genetic base for such differences. As well, by analyzing the distribution of breeding bird species, Abades and Marquet, characterize ecological phenomena. Based on statistical analyses of bird distribution, they propose that differences may be related to macroecological behavior.

Finally, in a novel discipline, Gonzalez-Nilo et al. present a review of a new sub-discipline termed nanobioinformatics. The article shows some examples of nanobiotechnology in life sciences, and discusses new challenges for bioinformatics and computational chemistry.

We hope this number of the Biological Research will be of interest for a broad number of biologists. We appreciate the energy and enthusiasm of the authors and other scientist that helped during the review process.

Christian GonZalez-Billault, Associate Editor, Biological Research

Nibaldo C. Inestrosa, Coordinator of the Frontiers of Science Program of the Chilean Academy of Sciences

Manuel J. SAntos, Editor in Chief, Biological Research 